\title{
Communication \\ Strategies to Optimize Adherence in Patients with Mycosis Fungoides
}

\author{
Warren H. Chan ${ }^{1, *}$, Daniel J. Lewis ${ }^{2}{ }^{\mathbb{C}}$, Madeleine Duvic ${ }^{3}$ and Steven R. Feldman ${ }^{1,4} \mathbb{C}$ \\ 1 Center for Dermatology Research, Department of Dermatology, Wake Forest School of Medicine, \\ Winston-Salem, NC 27157, USA; sfeldman@wakehealth.edu \\ 2 Department of Dermatology, University of Pennsylvania, Philadelphia, PA 19104, USA; \\ Daniel.lewis@pennmedicine.upenn.edu \\ 3 Department of Dermatology, MD Anderson Cancer Center, University of Texas, Houston, TX 77030, USA; \\ mduvic@mdanderson.org \\ 4 Department of Pathology, Wake Forest School of Medicine, Winston-Salem, NC 27104, USA \\ * Correspondence: warren.chan@bcm.edu; Tel.: +1-832-278-5398
}

check for updates

Citation: Chan, W.H.; Lewis, D.J.; Duvic, M.; Feldman, S.R. Strategies to Optimize Adherence in Patients with Mycosis Fungoides. Cells 2022, 11, 113. https://doi.org/10.3390/ cells11010113

Academic Editors: Ali R. Jazirehi and Pier Paolo Piccaluga

Received: 18 November 2021

Accepted: 28 December 2021

Published: 30 December 2021

Publisher's Note: MDPI stays neutral with regard to jurisdictional claims in published maps and institutional affiliations.

Copyright: (c) 2021 by the authors Licensee MDPI, Basel, Switzerland. This article is an open access article distributed under the terms and conditions of the Creative Commons Attribution (CC BY) license (https:// creativecommons.org/licenses/by/ $4.0 /)$.

\begin{abstract}
Patient adherence to medications for common skin conditions has been extensively studied over the past two decades, and suboptimal adherence is a primary contributor to treatment failure. The impact of sub-par adherence in cutaneous T-cell lymphoma (CTCL) patients has been largely unexplored, and promoting adherence in this patient population may represent a promising area of consideration for improving treatment outcomes. We apply patient adherence strategies that have been studied in dermatology to CTCL and provide concrete examples of how these strategies can be used to improve adherence in the CTCL setting. Through the implementation of small changes in how we present and counsel about therapeutic options to our patients, we can maximize patient adherence, which has the potential to optimize therapy regimens and reduce treatment failure.
\end{abstract}

Keywords: adherence; cutaneous T-cell lymphoma; anchoring; framing; loss aversion; mycosis fungoides; topical steroids; psoriasis; interferon; nitrogen mustard; mechlorethamine; brentuximab vedotin; mogamulizumab; romidepsin; vorinostat; PUVA; patient adherence

Patient adherence to medications for common skin conditions has been extensively studied over the past two decades and is a primary reason for treatment failure [1]. Having a serious condition, even acute leukemia, does not imply reliable adherence to treatment [2]. Adherence in cutaneous T-cell lymphoma (CTCL) is largely unexplored and may be an exciting target for improving treatment outcomes.

Mycosis fungoides (MF) is the most common form of CTCL; at the initial diagnosis, $70 \%$ of patients have early-stage disease (IA-IIA), for which the application of topical therapies, generally more difficult than taking oral medications, is often recommended [3]. With topical steroids alone, $63 \%$ of patients with $\mathrm{T} 1$ (patches or plaques covering $<10 \%$ body surface area [BSA]) disease and $25 \%$ with $\mathrm{T} 2$ (patches or plaques covering $\geq 10 \%$ BSA) disease achieve a complete response [4]. The difference in response rate may be at least partly attributable to lower adherence when application is needed to a larger area. Progression at five years is $10 \%$ in patients with T1 disease, $22 \%$ for T2, $48 \%$ for T3, and $56 \%$ for T4 [5]. Therefore, might we bring the rate of progression of those with T2 disease closer to those with T1, and decrease that in all stages, by improved adherence to treatment, whether topical or systemic? Patient adherence represents an area of untapped potential for improving treatment outcomes in CTCL.

Strategies to promote adherence have been extensively studied in psoriasis and can be applied to CTCL (Table 1) [1,6]. We employ the pyramid model for improving adherence: (1) building a foundation centered on trust and accountability, (2) addressing practical issues to make treatment as easy as possible for our patients, and (3) using behavioral 
techniques to give our patients an extra nudge to use their medications. We build trust by showing that we care (Table 1). More frequent office visits, scheduling a follow-up visit shortly after a new treatment is started, and asking patients to call or email us to report how their medication is working can build accountability. Forming good habits related to medication use in the short term may also promote better long-term use of treatment.

Next, we should make treatment as easy as possible for the patient. Can a patient on multiple topical steroids or multiple topical agents use only one instead? It may be that the reduction in adherence from adding a topical agent is not worth the marginal benefit conferred. When used consistently, one topical agent may be enough. If multiple agents are required, written patient instructions are likely to be needed. Patients tend to prefer foams and solutions over other vehicles [7]. We are taught that ointments are more potent than foams or solutions; however, ointments have zero potency if they are not used (Table 1). Patients are more likely to continue using their medication when they see that it is working; since it may take three weeks for CTCL patients to notice the difference from their treatment, asking them to call or email us after three days and then once per week for at least three weeks may build early accountability and lead to an earlier noticeable benefit, encouraging future adherence. For a similar reason, selecting fast-acting agents is helpful. Late-stage, severe, or long-standing CTCL can be depressing to patients and reduce adherence. Local radiation therapy and total skin electron beam therapy can clear even disseminated tumors and Sézary Syndrome for at least a few months, providing hope, and potentially improving adherence to future therapy and maintenance regimens. An important step involves setting expectations, as no treatments work overnight. Incorporating social work to help patients more easily navigate the treatment process and using institutional specialty pharmacies to aid in regularly scheduled calls to assess adherence are also recommended.

Lastly, we can employ behavioral techniques to nudge our patients in the right direction. Anchoring involves presenting an idea that is less desirable first to "anchor" the patient, making the next idea more desirable in comparison. For example, if we are recommending interferon to a patient, we might say, "Interferon is like insulin; it is given by injection. You are familiar with how patients with diabetes give themselves insulin injections twice a day, right? Well, this medication is not exactly like insulin-you only need to take the medication twice per week." Another behavioral technique involves the observation that humans tend to gravitate towards, and base their therapy decisions on, salient anecdotes more often than data. We can use saliency to our advantage by saying, "One of my other patients who reminds me of you and had disease very similar to yours had an excellent response to this medication. In fact, I think I saw that patient in this same exam room!" When counseling about adverse effects, patients tend to focus on the small risk of a side effect, rather than the comparatively large benefits of therapy. We should help reframe the patient's perspective by stating that 99 out of 100 patients do not have a certain side effect with a medication, rather than, for example, that mechlorethamine gel carries a $1 \%$ risk of nonmelanoma skin cancer. We can also use side effects to our advantage: the skin irritation from topical retinoids is "a sign that the drug is working."

If better adherence can contribute to a decreased progression risk, it may, for many CTCL patients, also prevent the need for systemic therapies, which are associated with high cost and side effects, which are major barriers to treatment in those with late-stage disease. Higher adherence to systemic therapies and adjuvant topical therapies may improve the patient's response to systemic therapies. Better adherence to topical steroids may lead to better control of pruritus. We might think that because of their cancer diagnosis, CTCL patients are more likely to adhere to treatment. However, chronicity and worsened impact of disease are associated with poorer adherence [8]. CTCL, especially MF, is not likely to be an exception. We should not assume that patient adherence is the norm and should instead work even harder to promote adherence in our cancer patients, especially given the severe consequences of non-adherence. 
Table 1. Patient adherence techniques and examples for the cutaneous T-cell lymphoma setting.

\begin{tabular}{ccc}
\hline Pyramid Level & Technique & Examples \\
\hline $\begin{array}{c}\text { Foundation of } \\
\text { trust and } \\
\text { accountability }\end{array}$ & Showing that we care & $\begin{array}{r}\text { Show up on time for clinic. Open the door to the exam room slowly to show that you are } \\
\text { not rushed. Do not look at your watch during the patient visit. }\end{array}$
\end{tabular}

Wash your hands in front of the patient.

Empathy: "I bet the previous treatments have been very frustrating, right?"

Assess patient satisfaction via surveys

Let the patient tell you their story without interrupting

Make yourself accessible. One method is to give your contact info to patients

More frequent office visits (increases white coat compliance)

Photopheresis involves two consecutive day sessions every $3-4$ weeks. If possible, have patients see a CTCL dermatologist before or after each session

Ask patients to call or email us to

report how the medication is working

\begin{tabular}{ccc}
\hline $\begin{array}{c}\text { Simplicity and } \\
\text { education }\end{array}$ & Simplify the treatment regimen & Switch multiple topical steroids or multiple topical agents to one steroid or topical agent \\
\hline Easier vehicle & Switch carumustine ointment to nitrogen mustard aqueous solution or \\
mechlorethamine gel
\end{tabular}

Shorten initial treatment interval

Since it takes roughly 3 weeks to see improvement, ask patients to call or email you in 3 days, then once per week for at least 3 weeks

Minimize cost of treatment

Use the EMR to auto-populate instructions for the pharmacist: "If the pharmacy offers a similar but less expensive option, feel free to switch to that medication, if the patient wants to."

Written instructions

Involve patients in the treatment choice

Choose a fast-acting agent

Printed from EMR, tear off pads, or sticky notes: "Hydrocortisone to face, triamcinolone to body, clobetasol to palms/soles"

Stage IA (T1): topical steroids v. topical mechlorethamine v. topical retinoids v. phototherapy v. imiquimod v. other

Stage IB (T2): bexarotene v. phototherapy v. interferon v. other

Total skin electron beam therapy can clear even disseminated tumors and Sezary Syndrome for at least a few months

Local radiation therapy is very effective at clearing discreet, even tumors

Behavioral

techniques

Anchoring

Interferon is like insulin; it is given by injection. You are familiar with how patients

with diabetes give themselves insulin injections twice a day, right? Well, this medication

is not exactly like insulin-you only need to take the medication twice per week."

Mogamulizumab: Say 4x/wk at first. Dosing actually starts weekly, then decreases to biweekly, then monthly

Use romidepsin $(3 \mathrm{x} / \mathrm{mo})$ or mogamulizumab (weekly at first) as anchors for brentuximab vedotin (once every $3 \mathrm{wks}$ )

Use vorinostat, which causes diarrhea, as an anchor for bexarotene, for which the side effects can be easily managed with pills for thyroid and cholesterol

Use PUVA (psoralen causes diarrhea) to anchor for nb-UVB, for which no extra pill is needed

Saliency

"One of our other patients who reminds me of you and had disease very similar to yours had an excellent response to this medication. In fact, I think I saw that patient in this same exam room."

Framing side effects

Nitrogen mustard has a 1-5\% increased risk of developing NMSCs. Reframe as “99/100 do not have this problem."

There is a $<0.01 \%$ chance of developing PML with BV. Reframe as "9999/10,000 do not have this problem."

Loss aversion (emphasize loss vs. gain)

Counteracting steroid phobia

Using side-effects to our advantage
"This drug can prevent your disease from growing worse"

"Steroids are cortisone medications, like over-the-counter hydrocortisone, only a little stronger. All-natural, organic, gluten-free"

Retinoids, imiquimod, resiquimod: "Skin irritation is a sign that it is working!" 
Author Contributions: Conceptualization, W.H.C., M.D. and S.R.F.; methodology, W.H.C., M.D. and S.R.F.; validation, W.H.C., D.J.L., M.D. and S.R.F.; investigation, W.H.C., D.J.L., M.D. and S.R.F.; resources, W.H.C., D.J.L., M.D. and S.R.F.; data curation, W.H.C., D.J.L., M.D. and S.R.F.; writingoriginal draft preparation, W.H.C., D.J.L., M.D. and S.R.F.; writing-review and editing, W.H.C., D.J.L., M.D. and S.R.F.; visualization, W.H.C., D.J.L., M.D. and S.R.F.; supervision, D.J.L., M.D. and S.R.F.; project administration, W.H.C. All authors have read and agreed to the published version of the manuscript.

Funding: This research received no external funding.

Conflicts of Interest: S.R.F. received consulting support from Helsinn. M.D. is the Blanche Bender Professor of Cancer Research and received support from the MDACC Stanton Endowment and MDACC Stanton CTCL Research Fund.

\section{References}

1. Lewis, D.J.; Feldman, S.R. Practical Ways to Improve Patient Adherence; CreateSpace Independent Publishing Platform: Scotts Valley, CA, USA, 2017; p. 194.

2. Hoppmann, A.L.; Chen, Y.; Landier, W.; Hageman, L.; Evans, W.E.; Wong, F.L.; Relling, M.V.; Bhatia, S. Individual prediction of nonadherence to oral mercaptopurine in children with acute lymphoblastic leukemia: Results from COG AALL03N1. Cancer 2021, 127, 3832-3839. [CrossRef] [PubMed]

3. Krejci-Manwaring, J.; McCarty, M.; Camacho, F.; Carroll, C.; Johnson, K.; Manuel, J.; Balkrishnan, R.; Hartle, J.; Fleischer, A.; Feldman, S. Adherence with topical treatment is poor compared with adherence with oral agents: Implications for effective clinical use of topical agents. J. Am. Acad. Dermatol. 2006, 54, S235-S236. [CrossRef] [PubMed]

4. Zackheim, H.; Kashani-Sabet, M.; Amin, S. Topical corticosteroids for mycosis fungoides. Experience in 79 patients. Arch. Dermatol. 1998, 134, 949-954. [CrossRef] [PubMed]

5. Kim, Y.; Liu, H.; Mraz-Gernhard, S.; Varghese, A.; Hoppe, R. Long-term outcome of 525 patients with mycosis fungoides and Sezary syndrome: Clinical prognostic factors and risk for disease progression. Arch. Dermatol. 2003, 139, 857-866. [CrossRef] [PubMed]

6. Woodbury, M.J.; Cohen, J.M.; Merola, J.F.; Perez-Chada, L.M. Leveraging behavioral economics to promote treatment adherence: A primer for the practicing dermatologist. J. Am. Acad. Dermatol. 2021, in press. [CrossRef]

7. Housman, T.; Mellen, B.; Rapp, S.; Fleischer, A.; Feldman, S. Patients with psoriasis prefer solution and foam vehicles: A quantitative assessment of vehicle preference. Cutis 2002, 70, 327-332. [PubMed]

8. Richards, H.; Fortune, D.; O'Sullivan, T.; Main, C.; Griffiths, C. Patients with psoriasis and their compliance with medication. J. Am. Acad. Dermatol. 1999, 41, 581-583. [PubMed] 